Case Report

\title{
Acute Rhabdomyolysis Associated with Coadministration of Levofloxacin and Simvastatin in a Patient with Normal Renal Function
}

\author{
Maria Paparoupa, Sebastian Pietrzak, and Adrian Gillissen \\ Department of Pulmonary Diseases, General Hospital Kassel, Kassel School of Medicine, Mönchebergstraße 41-43, \\ 53125 Kassel, Germany
}

Correspondence should be addressed to Maria Paparoupa; maria.paparoupa@yahoo.com

Received 8 May 2014; Accepted 11 July 2014; Published 22 July 2014

Academic Editor: Gerald S. Supinski

Copyright (C) 2014 Maria Paparoupa et al. This is an open access article distributed under the Creative Commons Attribution License, which permits unrestricted use, distribution, and reproduction in any medium, provided the original work is properly cited.

\begin{abstract}
We report a rare case of severe acute rhabdomyolysis in association with coadministration of levofloxacin and simvastatin in a patient with normal renal function. A 70-year-old Caucasian male was treated due to community acquired pneumonia with levofloxacin in a dosage of $500 \mathrm{mg}$ once and then twice a day. On the 8th day of hospitalization the patient presented with acute severe rhabdomyolysis requiring an intensive care support. After discontinuation of levofloxacin and concomitant medication with simvastatin $80 \mathrm{mg} /$ day, clinical and laboratory effects were totally reversible. Up to now, levofloxacin has been reported to induce rhabdomyolysis mainly in patients with impaired renal function, as the medication has a predominant renal elimination. In our case renal function remained normal during the severe clinical course. According to a recent case report rhabdomyolysis was observed due to interaction of simvastatin and ciprofloxacin. To our best knowledge this is the first case of interaction between simvastatin and levofloxacin to be reported. This case emphasizes the need of close monitoring of creatine kinase in patients under more than one potentially myotoxic medication especially when patients develop muscle weakness.
\end{abstract}

\section{Introduction}

Acute rhabdomyolysis is well-known as a clinical and laboratory syndrome associated with myoglobinuria, electrolyte disorders, and acute renal failure. Its diagnosis is based on the elevated creatine kinase levels after exclusion of myocardial infarction. Although initially described to be induced by crush injury or trauma, more common causes in hospitalized patients include coadministration of interacting drug agents [1]. The identification of a drug-induced rhabdomyolysis is important because the adverse effect is usually reversible after withdrawal of the suspected compound.

Particularly statins are well-known for their myotoxic potential especially in genetically susceptible individuals [2]. The symptoms of the myotoxic effect vary from simple myalgia to severe rhabdomyolysis [3]. Rhabdomyolysis caused by statins is mainly observed at the beginning of the treatment, after changing the agent [4], or even after increasing the dose of the subscribed medication [5]. The mean onset of myopathic side effects is approximately 6 months after initiation of treatment, although symptoms can occur at any time during the statin therapy [6].

Levofloxacin is a widely prescribed antibiotic for the treatment of community acquired pneumonia. It has a predominant renal elimination and dose reduction is necessary in patients with renal dysfunction [7]. Acute rhabdomyolysis due to Levofloxacin has been previously reported in elderly patients [8], in patients under hemodialysis [9], or after renal transplantation [10]. We report a rare case of severe acute rhabdomyolysis associated with coadministration of levofloxacin and simvastatin in a 70-year-old Caucasian male with normal renal function.

\section{Case Presentation}

A 70-year-old Caucasian male with previous medical history of coronary artery disease, hypertension, hyperlipidemia, 
and atrial fibrillation was presented with persistent dyspnea and cough for several days. An externally performed chest X-ray revealed consolidation of the upper lobe of the left lung. Having signs of community acquired pneumonia ampicillin/sulbactam was commenced and after developing diarrhea the antibiotic regime was changed to intravenous levofloxacin $500 \mathrm{mg} /$ day. After 5 days of consecutive treatment respiratory symptoms remained unchanged and the patient was subsequently admitted to our institution for further diagnostic procedures.

At the day of admission a clinical work up revealed reduced general health but no further clinical abnormalities. $\mathrm{He}$ denied back and joint pain or skin rashes. Routine laboratory exams were performed and have shown elevated liver (aspartate aminotransferase [ASAT] $129 \mathrm{U} / \mathrm{L}$, reference range 5-34 U/L and alanine aminotransferase [ALAT] $113 \mathrm{U} / \mathrm{L}$, reference $<55 \mathrm{U} / \mathrm{L}$ ) and muscle enzymes (creatine kinase [CK] $2521 \mathrm{U} / \mathrm{L}$, reference range 30-200 U/L) without signs of myocardial injury (quotient CKMB/CKMM 2). As the inflammatory markers were still significantly high (Creactive protein [CRP] $182 \mathrm{mg} / \mathrm{L}$, reference $<5 \mathrm{mg} / \mathrm{L}$ ) and the renal function was intact (blood potassium $4.10 \mathrm{mmol} / \mathrm{L}$ and creatinine concentrations $0.8 \mathrm{mg} / \mathrm{dL}$, resp.) levofloxacin therapy has been continued in a higher dosage (500 $\mathrm{mg} / 2 *$ day). Serum brain natriuretic peptide was normal, ruling out cardiogenic dyspnea. Active medication including simvastatin $80 \mathrm{mg} /$ day, metoprolol $25 \mathrm{mg} / 2 *$ day, aspirin $100 \mathrm{mg} /$ day, digitoxin $0,07 \mathrm{mg} /$ day, and furosemide $40 \mathrm{mg} /$ day was maintained. About 20 days before the admission of the patient to our department a former dose of simvastatin $40 \mathrm{mg} /$ day was doubled to $80 \mathrm{mg} /$ day as referred from his general physician. The reason for increasing the simvastatin dose was a persistent hypercholesterolemia despite the intake of the former antilipid therapy.

On the fourth day of hospitalization (overall day 8 of levofloxacin therapy) the patient complained about progressively increased bilateral legs and arms weakness involving both distal and proximal muscle groups. He further reported difficulty to stand up from his bed, dress up, or even use his pen to write. There was no bowel or bladder incontinence or further neurological symptoms. Physical examination revealed diffuse extremity weakness but no other neurological findings or signs of dermatomyositis. At this time muscle enzymes ([CK] $31539 \mathrm{U} / \mathrm{L})$ and parameters of liver toxicity ([ASAT] 486 U/L and [ALAT] $182 \mathrm{U} / \mathrm{L}$ ) increased dramatically indicating a rhabdomyolysis and the Patient was immediately treated with intravenous crystalloid hypotonic solution $(100 \mathrm{~mL} / \mathrm{h})$. We also proceded to discontinuation of simvastatin and levofloxacin, urine alkalinization and physical therapy.

A remarkable increase of muscle enzymes during the next days (maximum [CK] $159.450 \mathrm{U} / \mathrm{L}$ ) and severe generalized weakness, including respiratory insufficiency, made the admission to the ICU necessary, for implementation of noninvasive ventilation. As the renal retention parameters remained normal no hemodialysis was performed. Wide range of serology markers excluded other causes of rhabdomyolysis. A computer tomography of the chest showed withdrawal of the infiltration, although a $2 \mathrm{~cm}$ large tumor

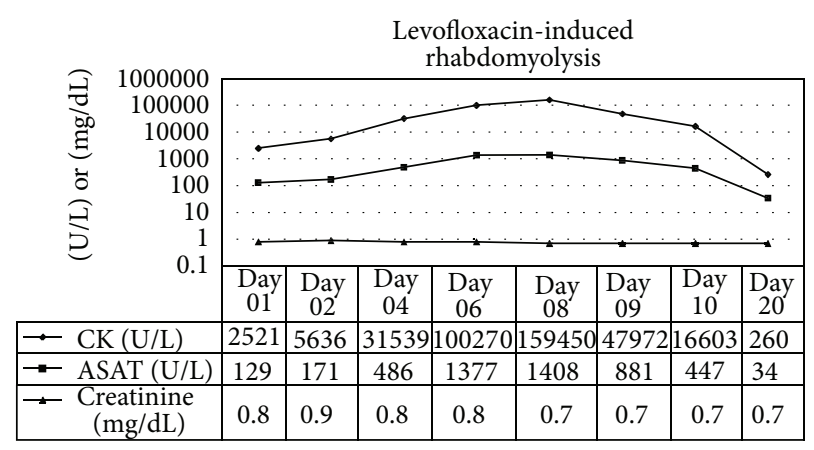

FIGURE 1: Serum creatinine, creatine kinase [CK], and aspartate aminotransferase [ASAT] during the severe clinical course. Notice the same curve morphology regarding the CK and ASAT evolution.

on the upper left lobe remained. A transbronchial biopsy confirmed the presence of nonsmall cell lung cancer.

Within a few days his symptoms improved significantly and his muscle and liver enzymes normalized (Figure 1). The patient was discharged at the 26th day of hospitalization with the diagnosis of iatrogenic rhabdomyolysis with acute hepatocellular damage. He presented again to the department of radiotherapy for the treatment of his lung carcinoma and his laboratory parameters were totally normal. The previous dosage of simvastatin $40 \mathrm{mg}$ /day was already resumed by his general physician.

\section{Discussion}

The diagnosis of acute severe rhabdomyolysis in our case is clear. The most probable cause for it is the treatment with levofloxacin, because there was a close temporal sequence between levofloxacin administration and the appearance of the symptoms, as well as clinical and laboratory recovery when the medication was discontinued. The adverse reaction appeared to be more severe when the dosage of levofloxacin was increased. Accordingly to Naranjo's probability scale a probable relationship between levofloxacin and rhabdomyolysis (total score 5) was confirmed even if simvastatin was considered to be an alternative cause of the adverse event (Table 1). Furthermore, all other potential reasons of muscle damage, such as Influenza $A$ and B virus [11], Parainfluenza type 1 and 2 virus [12], Coxsackie virus [13], HIV 1-2 virus [14], endocrinological abnormalities [15], and autoimmune myositis could be serologically excluded. Serum and urinary antigen and antibodies exams excluded Legionella pneumophila, Mycoplasma pneumoniae, Chlamydia pneumoniae, and Streptococcus pneumoniae as potential pathogens of the community acquired pneumonia.

Levofloxacin has a predominant renal elimination and a dose reduction is necessary in patients with impaired renal function [7]. Acute rhabdomyolysis has been previously reported in patients under hemodialysis [9] or after renal transplantation [10]. Petitjeans et al. described in 2003 a case of an elderly patient with severe rhabdomyolysis, suffering from acute renal failure [8]. In our case renal function was normal at the beginning of the therapy and remained intact 
TABLE 1: Naranjo's probability scale for our case.

\begin{tabular}{|c|c|c|}
\hline Naranjo's algorithm questions & Answer & Score \\
\hline $\begin{array}{l}\text { (1) Are there previous conclusive reports } \\
\text { of this reaction? }\end{array}$ & Yes & +1 \\
\hline $\begin{array}{l}\text { (2) Did the adverse event appear when } \\
\text { the drug was administered? }\end{array}$ & Yes & +2 \\
\hline $\begin{array}{l}\text { (3) Did the adverse reaction improve } \\
\text { when the drug was discontinued or a } \\
\text { specific antagonist was administered? }\end{array}$ & Yes & +1 \\
\hline $\begin{array}{l}\text { (4) Did the adverse reaction reappear } \\
\text { when the drug was readministered? }\end{array}$ & Do not know & 0 \\
\hline $\begin{array}{l}\text { (5) Are there alternative causes (or other } \\
\text { drugs) that could on their own explain } \\
\text { the adverse reaction? }\end{array}$ & Yes & -1 \\
\hline $\begin{array}{l}\text { (6) Did the adverse reaction reappear } \\
\text { when a placebo was given? }\end{array}$ & No & +1 \\
\hline $\begin{array}{l}\text { (7) Was the drug detected in the blood } \\
\text { (or other fluids) in concentrations known } \\
\text { to be toxic? }\end{array}$ & No & 0 \\
\hline $\begin{array}{l}\text { (8) Was the reaction more severe when } \\
\text { the dose was increased or less severe } \\
\text { when the dose was decreased? }\end{array}$ & Yes & +1 \\
\hline $\begin{array}{l}\text { (9) Did the patient have a similar reaction } \\
\text { to the same or similar drugs in any } \\
\text { previous exposure? }\end{array}$ & Do not know & 0 \\
\hline $\begin{array}{l}\text { (10) Was the adverse event confirmed by } \\
\text { any objective evidence? }\end{array}$ & No & 0 \\
\hline & Total score: & 5 \\
\hline \multicolumn{3}{|c|}{$\begin{array}{l}\text { The total score calculated from this table defines the category to which an } \\
\text { adverse event belongs to. } \\
\text { The categories are defined as shown below: } \\
\text { definite (certain): }>8 \text {; } \\
\text { probable: total score 5-8; } \\
\text { possible: total score 1-4; } \\
\text { doubtful (unlikely): total score }<1 \text {. }\end{array}$} \\
\hline
\end{tabular}

all along the severe clinical course, indicating that the adverse event is also probable in patients without renal impairment. An ofloxacin-induced rhabdomyolysis in previously healthy individuals has been reported from Hsiao et al. in 2005 [16], while other fluoroquinolones may also lead to this very rare adverse event mostly associated with acute kidney injury [17]. The histological effect of fluoroquinolones on the muscle cell has been demonstrated several years ago in juvenile rats, where no necrosis or lysis was seen, but the muscle fibers became atrophic [18].

The role of simvastatin in our case has to be also considered, as the medication has a very well confirmed myotoxicity especially when coadministrated with antibiotics, such as macrolides [19], fusidic acid [20], and azole antifungals [21]. According to a recent case report rhabdomyolysis was observed due to interaction of simvastatin and ciprofloxacin [22]. To the best of our knowledge, this is the first case of interaction between simvastatin and levofloxacin to be reported. Simvastatin is metabolized mainly in the liver by cytochrome CYP 3A4 and its active metabolite simvastatin acid is metabolized by cytochrome CYP 2C8 [23]. Levofloxacin is a weak inhibitor of cytochrome CYP $1 \mathrm{~A} 2$ and has no effect on cytochromes CYP 3A4 and CYP 2C 8 . As approximately $80 \%$ of levofloxacin is eliminated as unchanged drug in the urine through glomerular filtration and tubular secretion and only minimal metabolism occurs with the formation of no active metabolites [24], most likely other mechanisms are involved in the drug interaction of our case. Statins are substrates and strong inhibitors of P-glycoprotein (P-gp), also known as MDR1, which is a cellular drug efflux-transporter, responsible for the bioavailability of lots of medications [25]. In vitro studies have shown that levofloxacin is a potent inhibitor of Pgp-mediated efflux system [26]. According to this knowledge, levofloxacin may have blocked the metabolism of simvastatin leading to increased toxicity.

\section{Conclusion}

Our case report highlights the importance of close monitoring in patients under more than one potentially myotoxic medication, especially when patients develop new muscle weakness, by checking the serum creatine kinase and performing liver function tests.

\section{Conflict of Interests}

The authors declare that there is no conflict of interests regarding the publication of this paper.

\section{References}

[1] J. L. Zimmerman and M. C. Shen, "Rhabdomyolysis," Chest, vol. 144, no. 3, pp. 1058-1065, 2013.

[2] M. Needham and F. L. Mastaglia, "Statin myotoxicity: a review of genetic susceptibility factors," Neuromuscular Disorders, vol. 24, no. 1, pp. 4-15, 2014.

[3] M. Tomaszewski, K. M. Stepien, J. Tomaszewska, and S. J. Czuczwar, "Statin-induced myopathies," Pharmacological Reports, vol. 63, no. 4, pp. 859-866, 2011.

[4] S. Khanna and W. C. Mundell, "Rhadbomyolysis associated with co-administration of danazol and lovastatin," British Journal of Clinical Pharmacology, vol. 72, no. 1, pp. 166-167, 2011.

[5] U. Tayal and R. Carroll, "Should anyone still be taking simvastatin $80 \mathrm{mg}$ ?” BMJ Case Reports, 2013.

[6] K. E. Hansen, J. P. Hildebrand, E. E. Ferguson, and J. H. Stein, "Outcomes in 45 patients with statin-associated myopathy," Archives of Internal Medicine, vol. 165, no. 22, pp. 2671-2676, 2005.

[7] D. Czock, C. Hüsig-Linde, A. Langhoff et al., "Pharmacokinetics of moxifloxacin and levofloxacin in intensive care unit patients who have acute renal failure and undergo extended daily dialysis," Clinical Journal of the American Society of Nephrology, vol. 1, no. 6, pp. 1263-1268, 2006.

[8] F. Petitjeans, J. Nadaud, J. P. Perez et al., "A case of rhabdomyolysis with fatal outcome after a treatment with levofloxacin," European Journal of Clinical Pharmacology, vol. 59, no. 10, pp. 779-780, 2003.

[9] A. Gupta, N. Guron, M. Harris, and R. Bell, "Levofloxacininduced rhabdomyolysis in a hemodialysis patient," Hemodialysis International, vol. 16, no. 1, pp. 101-103, 2012.

[10] A. Korzets, U. Gafter, D. Dicker, M. Herman, and Y. Ori, "Levofloxacin and rhabdomyolysis in a renal transplant patient," 
Nephrology Dialysis Transplantation, vol. 21, no. 11, pp. 33043305, 2006.

[11] R. A. Fearnley, S. W. Lines, A. J. P. Lewington, and A. R. Bodenham, "Influenza A-induced rhabdomyolysis and acute kidney injury complicated by posterior reversible encephalopathy syndrome," Anaesthesia, vol. 66, no. 8, pp. 738-742, 2011.

[12] M. Douvoyiannis, J. M. Kielbasa, G. M. Chandrasekharan, C. L. Holmes, and M. R. Gomez, "Rhabdomyolysis associated with parainfluenza virus," Case Reports in Infectious Diseases, vol. 2013, Article ID 650965, 4 pages, 2013.

[13] Y. Wang, Y. Zhang, and Z. Ye, "Rhabdomyolysis following recent severe coxsackie virus infection in patient with chronic renal failure: one case report and a review of the literature," Renal Failure, vol. 28, no. 1, pp. 89-93, 2006.

[14] M. Douvoyiannis and N. Litman, "Acute encephalopathy and multi-organ involvement with rhabdomyolysis during primary HIV infection," International Journal of Infectious Diseases, vol. 13, no. 5, pp. e299-e304, 2009.

[15] M. R. Ardalan, K. Ghabili, R. Mirnour, and M. M. Shoja, "Hypothyroidism-induced rhabdomyolysis and renal failure," Renal Failure, vol. 33, no. 5, pp. 553-554, 2011.

[16] S. Hsiao, C. Chang, C. Tsao, Y. J. Lee, M. Hsu, and T. Wu, "Acute rhabdomyolysis associated with ofloxacin/levofloxacin therapy," Annals of Pharmacotherapy, vol. 39, no. 1, pp. 146-149, 2005.

[17] Q. Qian, S. H. Nasr, C. O. Akogyeram, and S. Sethi, "Myoglobinassociated acute kidney injury in the setting of ciprofloxacin administration," The American Journal of Kidney Diseases, vol. 59, no. 3, pp. 462-466, 2012.

[18] Y. Kashida and M. Kato, "Toxic effects of quinolone antibacterial agents on the musculoskeletal system in juvenile rats," Toxicologic Pathology, vol. 25, no. 6, pp. 635-643, 1997.

[19] J. Wagner, C. Suessmair, and H. W. Pfister, "Rhabdomyolysis caused by co-medication with simvastatin and clarithromycin," Journal of Neurology, vol. 256, no. 7, pp. 1182-1183, 2009.

[20] A. J. Burtenshaw, G. Sellors, and R. Downing, "Presumed interaction of fusidic acid with simvastatin," Anaesthesia, vol. 63, no. 6, pp. 656-658, 2008.

[21] M. A. Omar and J. P. Wilson, "FDA adverse event reports on statin-associated rhabdomyolysis," Annals of Pharmacotherapy, vol. 36, no. 2, pp. 288-295, 2002.

[22] R. D. Sawant, "Rhavdomyolysis due to an uncommon interaction of ciprofloxacin with simvastatin," Canadian Journal of Clinical Pharmacology, vol. 16, no. 1, pp. e78-e79, 2009.

[23] P. J. Neuvonen, M. Niemi, and J. T. Backman, "Drug interactions with lipid-lowering drugs: mechanisms and clinical relevance," Clinical Pharmacology and Therapeutics, vol. 80, no. 6, pp. 565581, 2006.

[24] D. N. Fish and A. T. Chow, "The clinical pharmacokinetics of levofloxacin," Clinical Pharmacokinetics, vol. 32, no. 2, pp. 101119, 1997.

[25] D. Williams and J. Feely, "Pharmacokinetic-pharmacodynamic drug interactions with HMG-CoA reductase inhibitors," Clinical Pharmacokinetics, vol. 41, no. 5, pp. 343-370, 2002.

[26] D. Pal and A. K. Mitra, "MDR- and CYP3A4-mediated drugdrug interactions," Journal of Neuroimmune Pharmacology, vol. 1, no. 3, pp. 323-339, 2006. 


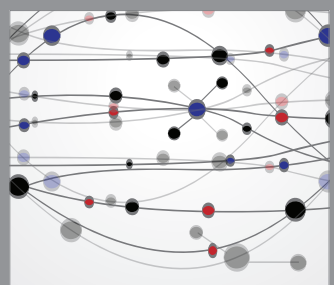

The Scientific World Journal
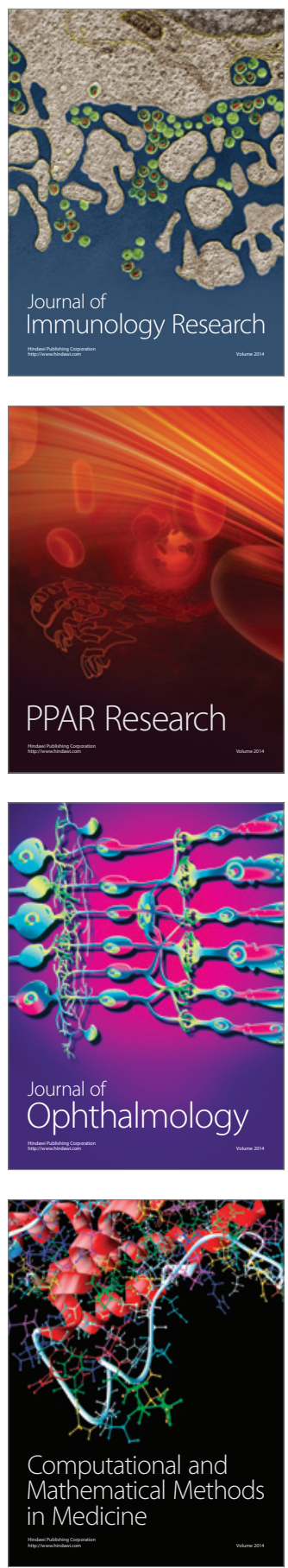

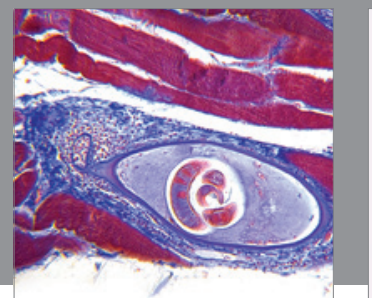

Gastroenterology

Research and Practice
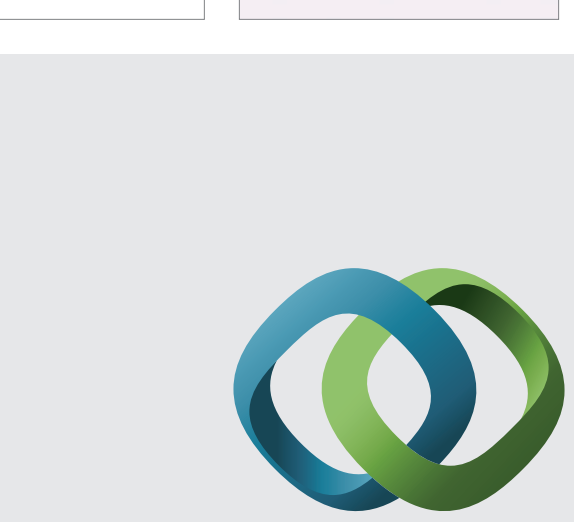

\section{Hindawi}

Submit your manuscripts at

http://www.hindawi.com
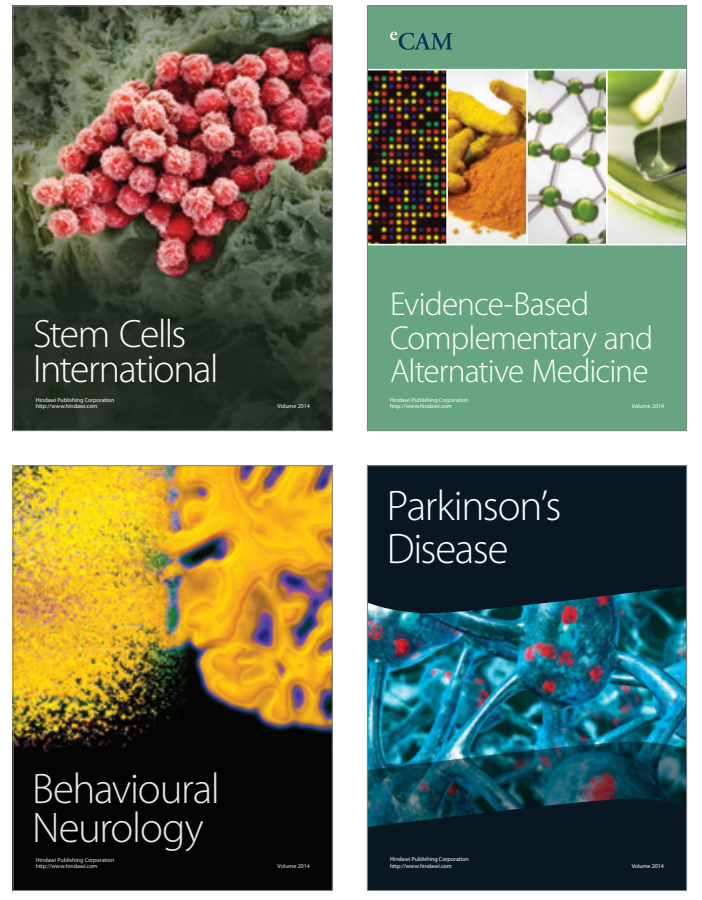
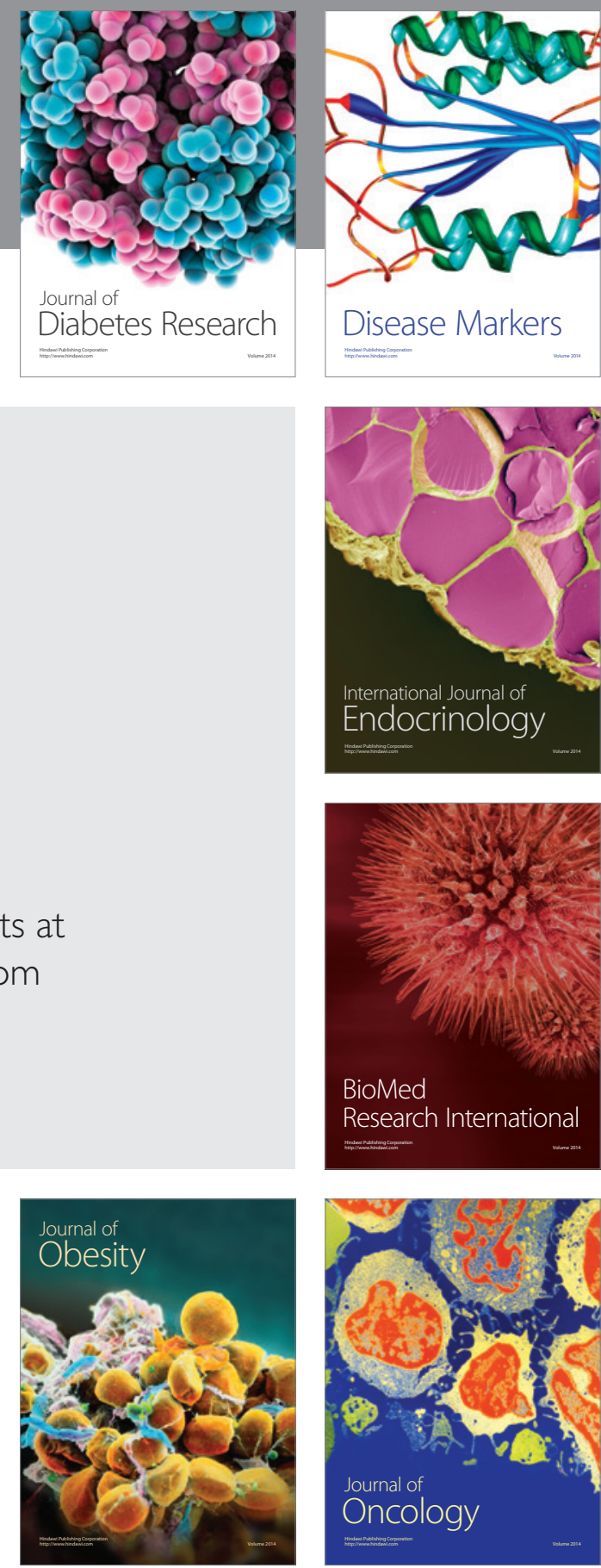

Disease Markers
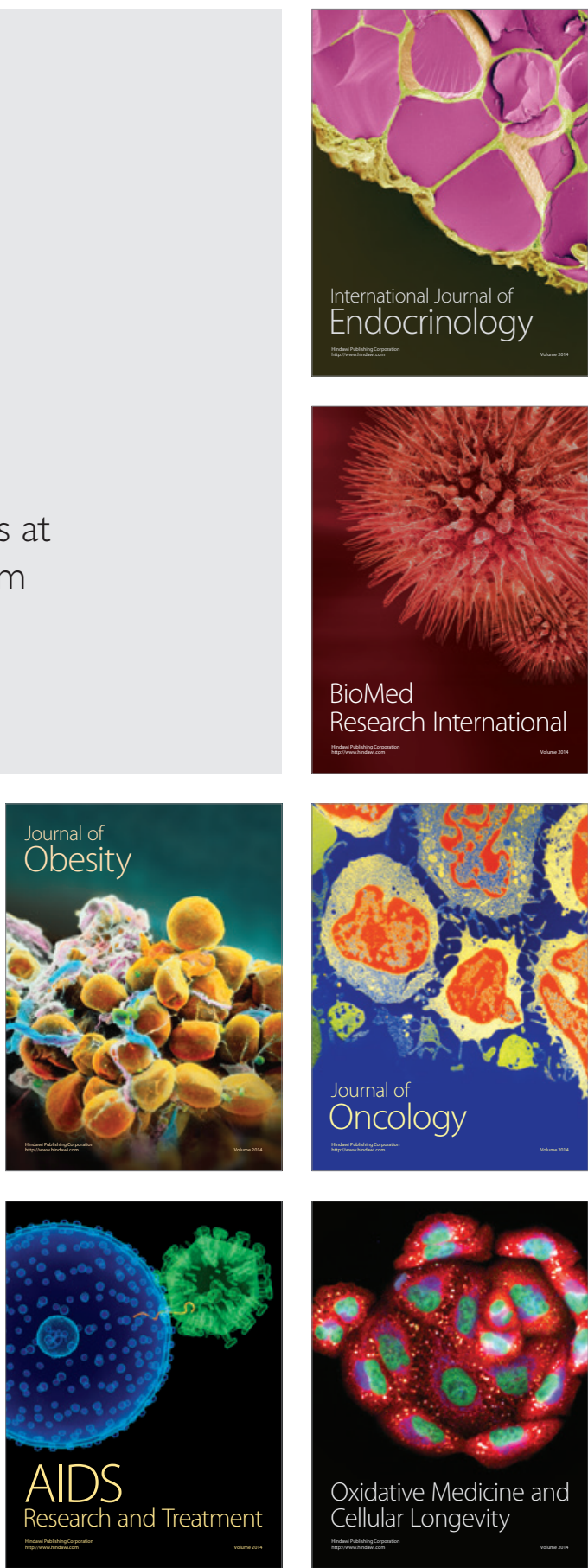